\title{
Interaction monitoring model of logo counseling website for college students' healthy self-esteem
}

\author{
Jacob Daan Engel ${ }^{1}$, Ventje Jeremias Lewi Engel ${ }^{2}$, Evangs Mailoa ${ }^{3}$ \\ ${ }^{1}$ Faculty of Theology, Satya Wacana Christian University, Indonesia \\ ${ }^{2}$ Department of Informatics, Harapan Bangsa Institute of Technology, Indonesia \\ ${ }^{3}$ Faculty of Information Technology, Satya Wacana Christian University, Indonesia
}

\begin{tabular}{l} 
Article Info \\
\hline Article history: \\
Received Feb 11, 2020 \\
Revised Jun 15, 2020 \\
Accepted Jul 23, 2020 \\
\hline Keywords: \\
Interaction monitoring model \\
Logo counseling \\
Logo counseling website \\
Low self-esteem \\
Machine learning
\end{tabular}

\begin{abstract}
The purpose of this research is to develop the client-counselor interaction monitoring model of the logo counseling website. The model attempts to help counselors in guiding and helping the students (clients) to achieve healthy self-esteem. Machine learning techniques integrated into the model will ensure that the recommendations can be available for counselors and supervisors in the near real-time environment. For the first implementation, a chatbot application is developed and tested with excellent responses from the students. Further research is needed to implement the complete specifications of the interaction monitoring model on the logo counseling website.
\end{abstract}

This is an open access article under the CC BY-SA license.

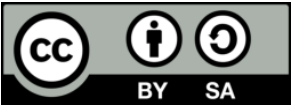

\section{Corresponding Author:}

Jacob Daan Engel,

Faculty of Theology,

Satya Wacana Christian University,

52-60 Diponegoro Street, Sidorejo, Salatiga 50711, Indonesia.

Email: jacob.engel@uksw.edu

\section{INTRODUCTION}

Logo counseling, a logotherapy modification, was developed to overcome the causative factors of low self-esteem as well as to discover meaning and purpose in the life of the students [1]. In addition, to overcome physical and psychological issues, the innovative model of logo counseling website was successfully designed with objectives and implemented with good responses from students [2]. However, field observations suggest that there is no mechanism for understanding or feeling the interaction between the client (student) and the counselor. Only after students pass a series of logo counselling sessions on the website can supervisors find out the results of the exit survey evaluation.From that fact, some thoughts arise: 1) Do students feel intimidated by the counselor in the website counseling logo process? 2) Do students tend to be more assertive, dynamic, or passive in the website counseling logo process? 3) How does the counselor build a conducive environment for students to express problems and emotional stress clearly? 4) How does the supervisor know the progress of the student's condition in real-time?

Correspondingly, Bambling revealed that counselors' role in information technology-based longdistance counseling is crucial, mainly due to the absence of face-to-face factors [3, 4]. The counselor's function in detecting the students' emotional state accurately in the online counseling process is essential [5]. Counselors can determine to what extent and in what ways the appropriate counseling approach is conducive to improving online communication. Efforts had been developed to monitor the client-counselor interactions [6]. However, the reasons for too many things to remember or the many forms to fill in make 
counselors reluctant to write comprehensive reports [6]. These issues and challenges also occured on the logo counseling website.

We think there needs to be a way to monitor the client-counselor interaction and at the same time help the counselor to better understand the emotional condition of the client/student. Therefore, we're trying to look at the advancement of machine learning methods in the artificial intelligence field. Machine learning techniques have been applied to mental health services in the communication dialog [7] and text-based chat application [8] to identify emotions. We believe that machine learning can assist client-counselor interaction monitoring inside the logo counseling website to ensure that counseling goals are met.

The problems and potentials described above are the motivation to develop a logo counseling website interaction monitoring model. The model aims to create a supportive environment where students express problems, accurately detect the emotional state of the students, and make sure the students' sustainability in the logo counseling process. The primary support in the model will be the use of information technology based on machine learning. This model is expected to be able to assist counselors and have a significant impact on clients to improve their quality of healthy self-esteem. This paper will therefore introduce a new monitoring model concept, which will evaluate the interaction between client and counselor on the logo counseling website.

Limitation of physical mobility, psychological pressure, and stigma from the community make students reluctant to seek counseling help [9, 10]. A survey found that 49 percent of young men are not too willing to discuss emotional problems for fear of people reacting negatively and judging [11]. On the other hand, remote counseling can provide counseling services to clients in their private environment [12, 13].

The use of information technology for counseling is more developed towards websites that can become a media intermediary between the client and his counselor without diminishing the meaning of the counseling process itself [10]. The primary purpose of implementing a website is to maximize the students' achievement by addressing their needs [14]. However, the problem is how to effectively implement counseling in the online environment $[14,15]$. Fortunately, an innovative model was developed to identify guidelines and features that contribute to the online counseling process [2]. This innovative model applied on the logo counseling website with features such as chat or instant messaging, email, and even audio conferencing between the counselor and the client [2].

The logo counseling website not only overcomes the client's limitations but also allows the counselor to reach clients without having to come in person. Counselor can also explore further sessions with client through features, such as notes in each session, session recording, and session replay [2]. The counselor can activate all or part of the features after obtaining the client's approval. Some examples of user interfaces and features of logo counseling website can be seen in this link: http://www.logokonseling.com/

On the other hand, the logo counseling website has limitations: 1) The logo counseling website is still entirely new, and its reliability can only be measured through an exit survey when the client completes a series of logo counseling sessions. We suspect that early indications and analysis can be done to effectively help the client to achieve his goals; 2) The effectiveness of counseling is based on open and honest communication between the client and the counselor. Working in a mere text-based environment on a website means no one can hear it. Misunderstandings are more likely to occur due to nonverbal gestures not being apparent in conversation. It is a question of how we can draw emotion from text-based communication to improve the interaction between the counselor and the client.

There are two main items must be done immediately based on the above explanation. First, measure the impact on client-counselor interactions resulting from the use of website technology. Not just the exit survey, but a proper evaluation should be done during the session and before it is completed. Second, develop a monitoring strategy or method on the logo counseling website to ensure therapeutic relationships between client and counselor. Website technology increases the impact on how counselors administer the counseling process. Counselors can provide better feedback according to client conditions; conversely, client expectations can be met near real-time. We believe the logo counseling website needs an advanced monitoring system which not only provides a conducive counseling environment but also at the same time gives recommendations to the counselor to better adapt his communication with the client.

Hansen showed that 5-10\% of clients in therapy and counseling experience a decline, and nearly $50 \%$ of clients experienced no significant changes during counseling sessions [16, 17]. Over time, the counselor does not always monitor the progress of the client's condition [6], but only goes through client sessions by following existing procedures. A monitoring mechanism on the continuity of client and counselor sessions while maintaining privacy and confidentiality is needed [18, 19]. The monitoring mechanism is conducted to firstly ensure that the counselor can maintain a conducive atmosphere during the session so that the student clearly and thoroughly explain the problem [18]. Secondly, checking and recording the student's progress. Third, providing feedback information to the counselor on client responses. 
Boswell explained that regular monitoring of sessions is necessary if therapeutic goals or treatments are to be achieved [20]. But, the biggest challenges of regular monitoring are time constraints, financial challenges, stakeholders' needs, and policy changes [20]. Eventually, this monitoring combined with evaluation mechanism can continously provide the conselor with feedback on the client's response and provide a summary at the end of each session. The summary will be input to the counselor for the next session's analysis material. Thus, client-counselor interaction monitoring can help counselors in directing, guiding, and analyzing client conditions [19]. This monitoring mechanism also needs to be implemented in website-based counseling.

In support of the online counseling monitoring, Bambling has revealed that the counselor's role in detecting the client's emotional state accurately in the online counseling process is crucial [5]. Counselors may determine the extent and in what ways appropriate counseling approaches can be used to enhance online communication. The role of counselors in IT-based long-distance counseling is crucial, mainly due to the lack of face-to-face factors [3, 4]. Efforts have been made to monitor interactions between clients and counselors $[21,22]$. However, the reasons for too many things to remember or the many forms to fill out make counselors reluctant to write a comprehensive report [6]. On the other hand, we believe that the development of machine learning techniques in the field of artificial intelligence can help with online mental health and counseling.

Machine learning techniques were applied to mental health services to analyze emotions in a conversation dialogue between clients and chatbots [7]. Detection of emotions and moods successfully applied to chat application using text pattern analysis [8] and smartphone sensing [23]. The classification of client problem topics using latent dirichlet-allocation (LDA) in crisis counseling helps counselors prepare themselves for clients [24]. Visualization of the conversation flows between clients and counselors with the support vector machine (SVM) algorithm successfully developed to assist supervisors in managing counselors [25]. Furthermore, a conversational agent was successfully designed to express empathy with corpus-based approach [26]. These studies showed the use of machine learning techniques to assist monitoring in the IT-based counseling and therapy process. Important point, the implementation of machine learning mentioned works well only when the system has access to data.

We see that the advantages of online counseling shine here. All interactions between client and counselor are completely recorded in digital form, whether in the form of text, audio, and video. This online counseling interaction data can help the machine to learn. Hence, the student's or client's problem-solving approach can be done based on evidence and is more quantitative than just qualitative estimates. There are four foundation aspects of interaction monitoring model of logo counseling website, as seen in Figure 1. They are client-counselor interaction, logo counseling website, session notes and records, and machine learning techniques.

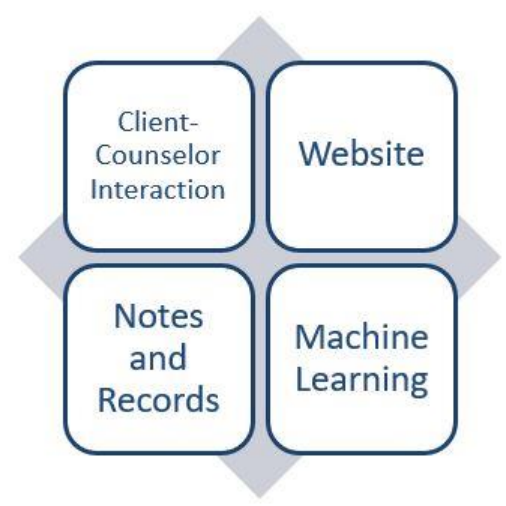

Figure 1. Four foundation aspects of interaction monitoring model

\section{RESEARCH METHOD}

This research used Design Science Research Methodology (DSRM) by Peffer because of its purpose to design and develop artifacts, such as constructs, models, or methods [27]. Design Science Research Methodology is almost the same as the R\&D methodology with the difference in its application. DSRM focuses on research related to human and computer interaction [28]. The stages of analysis and development of a client-counselor interaction monitoring model in the logo counseling website were adopted from DSRM. 
The research method we use consists of five steps: 1) Identify Problem and Motivations, we determine the problems that arise and motivations to solve those problems. The main problem we try to solve is how to better help counselors in guiding and helping the client throughout the online logo counseling processes. 2) Define The Objectives, we conclude the purpose of the solution to the problem that arises. The purpose of the proposed solution is a model that can be applied to read and analyze data from the online counseling process to monitor client-counselor interaction and create recommendations. 3) Design and Develop, we specify the design of the model to solve the problem and then develop the model. The model will integrate interaction data, objectives, and machine learning techniques. 4) Demonstration, we develop simulations for the model that have been made to solve the problems. The model applied to logo counseling website. 5) Evaluation, we evaluate the model that has been applied.

\section{RESULTS AND DISCUSSION}

\subsection{Model and specifications}

To design the interaction monitoring model for the logo counseling website, we identify interactions between client-counselor that can occur in the logo counseling website. The logo counseling website has three types of interaction: 1) Counselor notes, containing the counselor's session notes relating to the development of the client's condition in each session; 2) Voice calls, voice recordings of the counselor's conversation with the client. This recording can be played back if the counselor needs to do a more in-depth analysis of the session. 3) Chat, the most widely used form of session. The logo counseling session in chat includes written conversations between the counselor and the client.

After the three data sources obtained, they need to be preprocessed, which consists of two stages, namely data cleaning and feature extraction. Data cleaning is about eliminating punctuation and unnecessary words, both voice and text. Feature extraction is to accentuate words and phrases that can help identify client sentiment, emotions, and development in the next stage. The extraction of features will be beneficial for the next phase, namely machine learning engine. The machine learning engine has three modules, which are sentiment analysis, emotion detection, and notes understanding. The outputs of the machine learning engine are recommendations for counselor and client's condition summary.

Evaluating the model, we will implement a post-survey filled by the client and evaluation from the supervisor. Post-survey is a questionnaire that is filled out by the client after each session and when all counseling logo processes are completed. The post-survey comes from the client's opinion of his condition and interactions with the counselor. The evaluation from the supervisor is based on the notes of the counseling session that has taken place. From the previous explanation, the client-counselor interaction monitoring model in the logo counseling website consists of five parts: inputs, preprocessing machine learning engine, recommendations, and evaluations. The model can be seen in Figure 2.

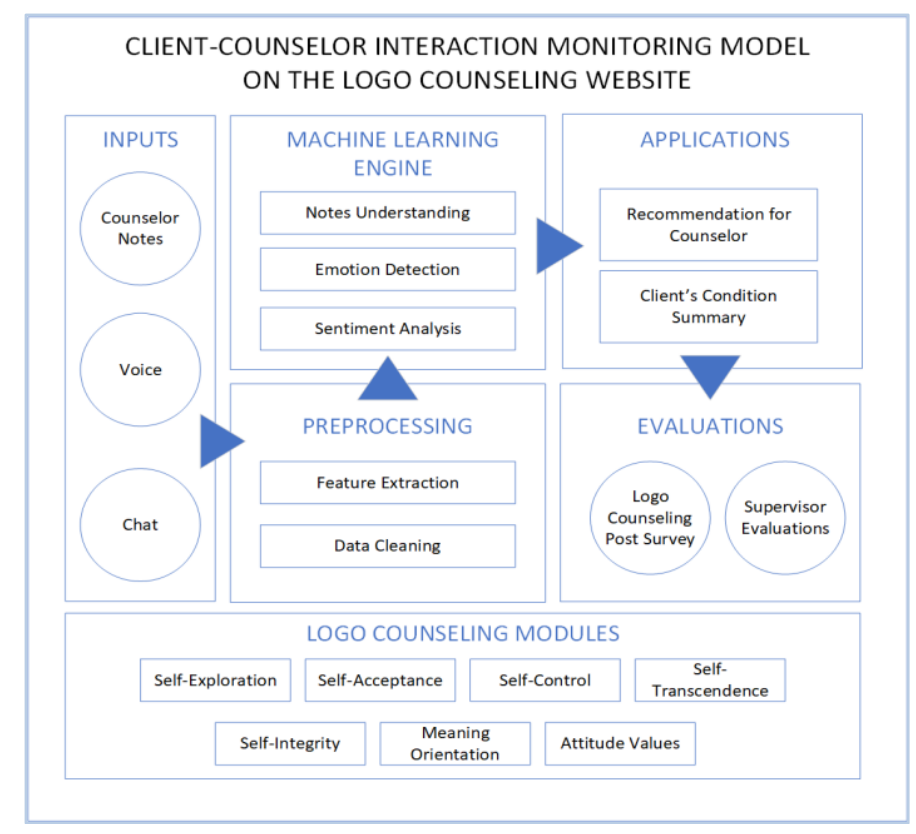

Figure 2. Client-counselor interaction monitoring model in logo counseling website 


\subsection{First implementation}

We developed a chat application to implement the model into an application that can be used and tested by clients and counselors. This chat application is a chatbot. This chatbot is used to determine profiles and the mental characteristics of clients. The purpose of this chat application is to demonstrate the feasibility of implementing the interaction monitoring model and to help clients deal more comfortably with problems in the session.

There are three stages in the general counseling process, namely: pre-assessment, session, and postassessment. Pre-assessment is the stage where the counselor attempts to understand problems and challenges facing the client. At this first stage, it isn't uncommon for counselor to experience problems. Meanwhile, teenagers and college students are more comfortable with text chatting for the initial stage of counseling, not video calling [29]. Gamification will make the client's counseling process made more enjoyable. Gamification is the process of transforming a series of activities into a game using gift-giving or game design [30]. In this implementation, machine learning is used to help gamification. To help gamification, we designed mascot with a youthful look and pre-assessment questions that are easier to understand by including illustrations. The client-counselor monitoring interaction model with machine learning was successfully implemented in a web chat application for pre-assessment, which would then be followed for online counseling sessions. We only focus on the pre-assessment phase and the beginning of logo counseling sessions.

Utilize machine learning, the chatbot application can display the result after client filled out the preassessment survey. We used the previous result research to determine the low self-esteem problem level of the client [31]. Then, the chatbot can connect the client with the approriate counselor based on the result. Nevertheless, for now, the counselor assignment process is carried out by round-robin system. If there are three counselors, then the assignment is 1-2-3, then back to counselor number 1. After the assignment, the counselor and client can make a schedule to start the logo counseling session. For this first implementation evaluation, we asked the clients to fill out eleven items of Yes-No questionnaires. The clients are college students between 18-22 years old already did the pre-assessment and started the logo counseling session via the website. There are fifteen clients asked. The results can be seen in Table 1 .

Table 1. Clients evaluation of interaction monitoing model in logo counseling website

\begin{tabular}{|c|c|c|c|}
\hline No. & Questions & Yes & No \\
\hline 1 & You find it difficult to seek help for counseling & $73.3 \%$ & $26.7 \%$ \\
\hline 2 & You feel embarrassed or afraid to go to counselor office & $93.3 \%$ & $6.7 \%$ \\
\hline 3 & Logo counseling website informs you with necessary informations about logo counseling & $86.7 \%$ & $13.3 \%$ \\
\hline 4 & Logo counseling website makes it easy for you to get counseling help & $86.7 \%$ & $13.3 \%$ \\
\hline 5 & Logo counseling pre-assessment via chatbot is helpful to you & $80 \%$ & $20 \%$ \\
\hline 6 & Logo counseling pre-assessment via chatbot does not intimidate you & $93.3 \%$ & $6.7 \%$ \\
\hline 7 & Logo counseling pre-assessment questions via chatbot are concise and clear to you & $86.7 \%$ & $13.3 \%$ \\
\hline 8 & You can easily view the result of pre-assessment via chatbot & $93.3 \%$ & $6.7 \%$ \\
\hline 9 & You find the start of the logo counseling session is helpful & $73.3 \%$ & $26.7 \%$ \\
\hline 10 & You want to continue the sessions in logo counseling website & $53.3 \%$ & $46.7 \%$ \\
\hline 11 & You want to recommend logo counseling website to your friends and/or family & $86.7 \%$ & $13.3 \%$ \\
\hline
\end{tabular}

The evaluation starts with verification about their difficulties in seeking help for counseling, with $73.3 \%$ felt challenging to get counseling help, and $93.3 \%$ felt embarrassed to go to the counselor office. Most of the clients find that information on the logo counseling website is clear and made them easy to get counseling help. Pre-assessment via chatbot left an excellent impression on clients. Besides the questionnaire, we also obtained comments from clients about pre-assessment via chatbot. In summary, they felt refreshing to try something new like chatbot with gamification. Most of them also found that the beginning of logo counseling sessions is helpful.

Despite the logo counseling website's uniqueness and strengths, only $53.3 \%$ wanted to continue the sessions. This result was puzzling us. Further analysis, we found that most of them afraid about being exposed if the hacker attacks the website. This security issue is homework for us to give a guarantee about security and data safety. Interestingly, $86.7 \%$ still recommended the logo counseling website service to friends and family. It is because we thought they found something new and refreshing about the preassessment phase. 


\section{CONCLUSION}

The client-counselor interaction monitoring model of the logo counseling website is a new idea that will help the counselor in practical terms and have a significant impact on client development. The model aims to create a conducive environment for students to express problems, accurately detect the emotional state of students, and ensure the students' sustainability in the logo counseling process. Machine learning techniques are incorporated in the model and the system. The first implementation is done as a chatbot application for pre-assessment of the client's mental health condition and the start of the logo counseling session. The responses are excellent, and the students found it helpful to start logo counseling sessions.

This model will add academic insights into the field of counseling and information technology to transform human interaction in the digital world. For future development, we will endeavor to implement the model with full scpecifications. Additionally, further research is needed to ensure that this model can eventually help counselors guide the client, supervisor assess the adherence to logo counseling best practices, and clients solve their low self-esteem problems. Some exciting venture for further research is to identify client's emotions criteria and to build a system to detect the client's emotion in the real-time environment.

\section{ACKNOWLEDGEMENTS}

We would like to thank you for the funding for this research. This work was supported by the Internal Research Development Grant provided by Research, Publications, and Community Development Body of Satya Wacana Christian University.

\section{REFERENCES}

[1] J. D. Engel and L. Loekmono, "Logo Counseling for Low Spiritual Self-Esteem Among College Students," Int. J. Eval. Res. Educ., vol. 7, no. 3, pp. 236-243, 2018.

[2] J. D. Engel, V. J. L. Engel, and E. Mailoa, "Innovative Model for Logo Counseling Website," TELKOMNIKA (Telecommunication Comput. Electron. Control ), vol. 16, no. 5, pp. 2111-2117, 2018.

[3] A. Kingsley and J. A. Henning, "Online and Phone Therapy: Challenges and Opportunities," J. Individ. Psychol., vol. 71, no. 2, pp. 185-194, 2015.

[4] D. Richards and N. Viganó, "Online Counseling: A Narrative and Critical Review of the Literature," J. Clin. Psychol., vol. 69, no. 9, pp. 994-1011, 2013.

[5] M. Bambling, R. King, W. Reid, and K. Wegner, "Online counselling: The experience of counsellors providing synchronous single-session counselling to young people," Couns. Psychother. Res., vol. 8, no. 2, pp. 110-116, 2008.

[6] L. Overington and G. Ionita, "Progress monitoring measures: A brief guide," Can. Psychol., vol. 53, no. 2, pp. 82-92, 2012 .

[7] K. J. Oh, D. Lee, B. Ko, and H. J. Choi, "A Chatbot for Psychiatric Counseling in Mental Healthcare Service Based on Emotional Dialogue Analysis and Sentence Generation," in Mobile Data Management (MDM), 2017 18th IEEE International Conference, pp. 371-375, 2017.

[8] M. Dheepthi and M. Hemalatha, "Dynamic Mood Detection in Chat Application Using Text Pattern Analysis," Int. J. Adv. Appl. Sci., vol. 4, no. 4, pp. 124-129, 2015.

[9] F. T. L. Leong, H. H. W. Kim, and A. Gupta, "Attitudes Toward Professional Counseling among Asian-American College Students: Acculturation, Conceptions of Mental Illness, and Loss of Face," Asian Am. J. Psychol., vol. 2, no. 2, pp. 140-153, 2011.

[10] Z. Ardi, M. R. M. Putra, and I. Ifdil, "Ethics And Legal Issues In Online Counseling Services: Counseling Principles Analysis," J. Psikol. Pendidik. dan Konseling, vol. 3, no. 2, p. 15, 2018.

[11] K. J. Glasheen and M. A. Campbell, "The use of online counselling within an Australian secondary school setting: A practitioner's viewpoint," Couns. Psychol. Rev., vol. 24, no. 2, pp. 42-51, 2009.

[12] C. R. McAdams III and K. L. Wyatt, "The Regulation of Technology-Assisted Distance Counseling and Supervision in the United States: An Analysis of Current Extent, Trends, and Implications," Couns. Educ. Superv., vol. 49, no. March, pp. 179-192, 2010.

[13] M. Dowling and D. Rickwood, "Online counseling and therapy for mental health problems: A systematic review of individual synchronous interventions using chat," J. Technol. Hum. Serv., vol. 31, no. 1, pp. 1-21, 2013.

[14] J. D. Engel and E. Mailoa, Website Based Counseling Logo (in Bahasa). Yogyakarta: Kanisius, 2017.

[15] K. D. Baker and M. Ray, "Online counseling: The good, the bad, and the possibilities," Couns. Psychol. Q., vol. 24, no. 4, pp. 341-346, 2011.

[16] W. N. W. Hashim, M. R. Othman, S. Madian, and M. I. Syafiq, "Development of a Usable Online Counseling Management System,” Procedia - Soc. Behav. Sci., vol. 97, no. 1, pp. 761-765, 2013.

[17] N. B. Hansen, M. J. Lambert, and E. M. Forman, "The psychotherapy dose-response effect and its implications for treatment delivery services," Clin. Psychol. Sci. Pract., vol. 9, no. 3, pp. 329-343, 2002.

[18] B. M. Ogles and M. J. Lambert, "Essentials of outcome assessment," Psychother. Res., vol. 19, no. 4-5, pp. 493-501, 2009.

[19] S. T. Meier, Incorporating Progress Monitoring and Outcome Assessment Into Counseling and Psychotherapy: A Primer. New York: Oxford University Press, 2015. 
[20] J. F. Boswell, D. R. Kraus, S. D. Miller, and M. J. Lambert, "Implementing routine outcome monitoring in clinical practice: Benefits, challenges, and solutions," Psychother. Res., vol. 25, no. 1, pp. 6-19, 2015.

[21] M. Tasma, E. J. Liemburg, H. Knegtering, P. A. E. G. Delespaul, A. Boonstra, and S. Castelein, "Exploring the use of Routine Outcome Monitoring in the treatment of patients with a psychotic disorder," Eur. Psychiatry, vol. 42, no. 1, pp. 89-94, 2017.

[22] D. G. Hays, “Introduction to Counseling Outcome Research and Evaluation,” Couns. Outcome Res. Eval., vol. 1, no. 1 , pp. 1-7, 2010.

[23] X. Zhang, W. Li, X. Chen, and S. Lu, "MoodExplorer: Towards Compound Emotion Detection via Smartphone Sensing," Proc. ACM Interactive, Mobile, Wearable Ubiquitous Technol., vol. 1, no. 4, pp. 1-30, 2018.

[24] K. Dinakar, J. Chen, H. Lieberman, R. Picard, and R. Filbin, "Mixed-Initiative Real-Time Topic Modeling \& Visualization for Crisis Counseling," in Proceedings of the 20th International Conference on Intelligent User Interfaces - IUI '15, pp. 417-426, 2015.

[25] Y. Hayashida, T. Uetsuji, Y. Ebara, and K. Koyamada, "Category classification of text data with machine learning technique for visualizing flow of conversation in counseling," in Proceedings - 2017 NICOGRAPH International, NICOInt 2017, pp. 37-40, 2017.

[26] R. R. Morris, K. Kouddous, R. Kshirsagar, and S. M. Schueller, "Towards an Artificially Empathic Conversational Agent for Mental Health Applications: System Design and User Perceptions," J. Med. Internet Res., vol. 20, no. 6, pp. 1-10, 2018.

[27] K. Peffers, T. Tuunanen, M. Rothenberger, and S. Chatterjee, "A Design Science Research Methodology for Information Systems Research,” J. Manag. Inf. Syst., vol. 24, no. 3, pp. 45-78, 2007.

[28] G. Fernandes, J. Barbosa, E. B. Pinto, M. Araújo, and R. J. Machado, "Applying a Method for Measuring the Performance of University-Industry R\&D Collaborations: Case Study Analysis," in Procedia Computer Science, vol. 164, pp. 424-432, 2019.

[29] S. Cipolletta and D. Mocellin, "Online counseling: An exploratory survey of Italian psychologists' attitudes towards new ways of interaction," Psychother. Res., vol. 28, no. 6, pp. 909-924, 2018.

[30] A. Shipepe and A. Peters, "Designing an interactive career guidance learning system using gamification," Proceedings of the Second African Conference for Human Computer Interaction: Thriving Communities, pp. 1-4, 2018.

[31] J. D. Engel, Basic Value of the Counseling Logo (in Bahasa). Yogyakarta: Kanisius, 2014. 